\title{
DETERMINAÇÃO DO DIÂMETRO DE PARTÍCULAS DE ESPÉCIES DE COBALTO EM CATALISADORES SUPORTADOS EM $\gamma$ ALUMINA
}

\author{
H. A. OLIVEIRA ${ }^{1}$ e F. B. PASSOS ${ }^{1}$ \\ ${ }^{1}$ Universidade Federal Fluminense, Departamento de Engenharia Química e Petróleo \\ E-mail para contato: fbpassos@vm.uff.br
}

\begin{abstract}
RESUMO - Catalisadores de cobalto suportados em alumina são usados em diferentes processos e a identificação e a dispersão das espécies de cobalto é um parâmetro fundamental para melhorar a seletividade e atividade catalítica. As técnicas de fissisorção, espectroscopia de energia dispersiva, espectroscopia de fotoelétrons de raios-X, difração de raios-X e redução à temperatura programada foram usadas para a caracterização de catalisadores contendo 1, 2, 3 e 4\% de cobalto suportados em $\gamma$-alumina. Foi verificado que o $\mathrm{CoAl}_{2} \mathrm{O}_{4}$ está altamente disperso em todas as amostras e, com o aumento do teor de cobalto nos catalisadores a fração de cobalto como $\mathrm{CoAl}_{2} \mathrm{O}_{4}$ diminui enquanto a fração de $\mathrm{CoO}$ e $\mathrm{Co}_{3} \mathrm{O}_{4}$ aumentam. $\mathrm{O}$ diâmetro dos cristalitos de $\mathrm{Co}_{3} \mathrm{O}_{4}$ foram 10 e $14 \mathrm{~nm}$ para os catalisadores com 3 e $4 \%$ de cobalto. Para os demais catalisadores, os cristalitos de $\mathrm{CoO}$ e $\mathrm{Co}_{3} \mathrm{O}_{4}$ não foram detectados por XRD. Contudo, foi possível estimar o tamanho médio das partículas de óxidos de cobalto $\left(\mathrm{CoO}\right.$ e $\left.\mathrm{Co}_{3} \mathrm{O}_{4}\right)$ por espectroscopia de fotoelétrons de raios-X. Para o catalisador $1 \mathrm{Co} / \mathrm{Al}_{2} \mathrm{O}_{3}$ as partículas de cobalto estão $100 \%$ dispersas, o catalisador $2 \mathrm{Co} / \mathrm{Al}_{2} \mathrm{O}_{3}$ apresentou diâmetro de partícula de $2 \mathrm{~nm}$ e os catalisadores $3 \mathrm{e}$ $4 \% \mathrm{Co} / \mathrm{Al}_{2} \mathrm{O}_{3}$ apresentaram $4 \mathrm{~nm}$.
\end{abstract}

\section{INTRODUÇÃO}

Nanotubos de carbono são uma classe de fibras descobertos em 1991 por Iijima (1991) e apresentam extraordinárias propriedades elétricas e mecânicas. Para produção dos nanotubos pela rota catalítica é necessária a obtenção de partículas metálicas de tamanho nanométrico (Cheung et al. 2002). Entre os catalisadores utilizados para síntese de nanotubos, os catalisadores de cobalto suportados em alumina apresentaram bom desempenho, principalmente para produção de nanotubos de parede única (Li et al. 2010). Para melhorar a dispersão das partículas normalmente é feita uma impregnação com baixo teor de cobalto em um suporte de elevada área específica como, por exemplo, a $\gamma$-alumina. Contudo, este baixo teor pode levar a formação de óxidos de cobalto de difícil redução que são inativos para síntese dos nanotubos (Zhang et al. 2003). Desta forma é necessário fazer uma caracterização detalhada para identificação e quantificação das espécies de cobalto que são capazes de produzir nanotubos de carbono. Para esta finalidade, as técnicas de fissisorção de $\mathrm{N}_{2}$, espectroscopia de energia dispersiva (EDX), espectroscopia de fotoelétrons (XPS), difração de raios-X (XRD) e redução à temperatura programada (TPR) foram usadas e, com os resultados, foi possível propor um esquema para a dispersão destas espécies em catalisadores suportados em alumina contendo 1, 2, 3 e 4\% de cobalto. 


\section{9 a 22 de outubro de 2014 \\ Florianópolis/SC}

\section{SEÇÃO EXPERIMENTAL}

O precursor do suporte (Catapal-A Bohemite) foi seco a temperatura de $120{ }^{\circ} \mathrm{C}$ por $16 \mathrm{~h} \mathrm{e}$, então, calcinado a temperatura de $550{ }^{\circ} \mathrm{C}$ por duas horas. Catalisadores de cobalto suportados em $\gamma$-alumina foram preparados por impregnação seca usando uma solução de $\mathrm{Co}\left(\mathrm{NO}_{3}\right)_{2} \cdot 6 \mathrm{H}_{2} \mathrm{O}$ (Merck). A concentração mássica de cobalto nos catalisadores foi de 1, 2, 3 e 4\%. As medidas de área específica, volume de poros e distribuição do tamanho dos poros foram medidas em um ASAP 2010 Micromeritics, por fisissorção de $\mathrm{N}_{2}$. As amostras foram evacuadas até $3 \mu \mathrm{m}$ de $\mathrm{Hg}$ a uma temperatura de $220^{\circ} \mathrm{C}$. O volume de poros e a distribuição de seu tamanho foram calculados pela curva de dessorção usando o método BJH. A composição química dos catalisadores foi determinada por um aparelho EDX-720 Shimadzu. As analises de XPS foram feitas em um ESCALAB 250Xi ThermoScientific com fonte monocromática de raios-X Al Ka. A pressão dentro da câmara de análise foi menor que $1 \times 10^{-9}$ mbar. Devido ao carregamento elétrico das amostras durante a análise foi feita uma compensação de carga por FloodGun e os picos foram corrigidos pela referência da linha $\mathrm{C} 1 \mathrm{~s}$ a $284,8 \mathrm{eV}$. A difração de raios-X dos catalisadores foi feita em um equipamento Rigaku Miniflex com radiação monocromática $\mathrm{Cu}-\mathrm{K} \alpha(1.540 \AA)$ com passo de 0.05 graus por min. $O$ diâmetro médio dos cristalitos de cobalto foram calculadas a partir da linha mais intensa $2 \theta=36,9^{\circ}$ usando a formula de Scherrer. A redução à temperatura programada foi realizada usando um espectrômetro de massas quadrupole (Omnistar, Balzers). As amostras foram secas em He até a temperatura de $150{ }^{\circ} \mathrm{C}$ antes de iniciar a redução. Após o resfriamento da temperatura até a temperatura de ambiente, uma mistura de $5 \% \mathrm{H}_{2} / \mathrm{Ar}$ foi introduzida no reator a uma vazão de $30 \mathrm{~mL} / \mathrm{min}$. A temperatura foi elevada a uma taxa de $10{ }^{\circ} \mathrm{C}$ por min até a temperatura de $1000{ }^{\circ} \mathrm{C}$.

\section{RESULTADOS E DISCUSSÃO}

As medidas de fisissorção foram feitas para determinar as mudanças na área especifica e distribuição dos poros dos catalisadores. A Tabela 1 mostra as propriedades texturais dos catalisadores e do suporte. Os valores da área específica e distribuição do diâmetro dos poros do suporte são típicos da fase gama. Os catalisadores com maiores quantidade de cobalto em sua composição apresentaram menor área específica. Este efeito pode ser atribuído ao bloqueio dos poros de menor diâmetro pelas partículas de óxido de cobalto como mostra a Figura 1(a). Isto leva a um aumento do valor médio do diâmetro dos poros como mostra a Tabela 1.

Tabela 1 - Valores de área específica e diâmetro médio dos poros obtidos por fisisssorção; fração mássica de cobalto dos catalisadores determinado por EDX e; fração mássica superficial de cobalto e fração de Co como $\mathrm{CoAl}_{2} \mathrm{O}_{4}$ dos catalisadores obtido por XPS.

\begin{tabular}{|c|c|c|c|c|c|}
\hline Amostra & $\begin{array}{c}\text { Área } \\
\text { específica } \\
\left(\mathrm{m}^{2} / \mathrm{g}\right)\end{array}$ & $\begin{array}{c}\text { Diâmetro } \\
\text { médio dos } \\
\text { poros }(\mathrm{nm})\end{array}$ & $\begin{array}{c}\text { Fração } \\
\text { mássica de } \\
\mathrm{Co}(\%)^{*}\end{array}$ & $\begin{array}{c}\text { Fração } \\
\text { superficial de } \\
\mathrm{Co}^{* *}\end{array}$ & $\begin{array}{c}\text { Fração Co } \\
\text { como }^{*} \\
\mathrm{CoAl}_{2} \mathrm{O}_{4}{ }^{* *}\end{array}$ \\
\hline $\mathrm{Al}_{2} \mathrm{O}_{3}$ & 282 & 7,1 & 0,00 & 0,00 & 0,00 \\
\hline $1 \mathrm{Co} / \mathrm{Al}_{2} \mathrm{O}_{3}$ & 266 & 7,4 & 0,92 & 0,96 & 0,93 \\
\hline $2 \mathrm{Co} / \mathrm{Al}_{2} \mathrm{O}_{3}$ & 257 & 7,5 & 2,02 & 0,47 & 0,82 \\
\hline $3 \mathrm{Co} / \mathrm{Al}_{2} \mathrm{O}_{3}$ & 256 & 7,5 & 2,99 & 0,49 & 0,69 \\
\hline $4 \mathrm{Co} / \mathrm{Al}_{2} \mathrm{O}_{3}$ & 248 & 7,8 & 3,96 & 0,64 & 0,52 \\
\hline
\end{tabular}




\section{9 a 22 de outubro de 2014 \\ Florianópolis/SC}
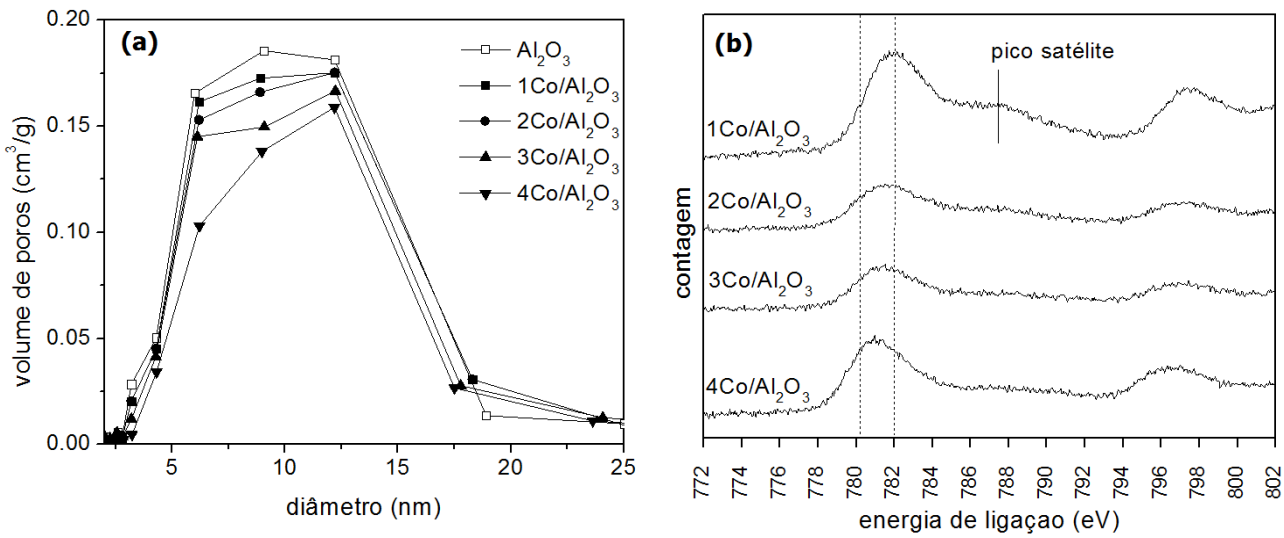

Figura 1-(a) Distribuição do diâmetro dos poros e (b) espectro de fotoelétrons na região Co2p

A composição de cobalto nos catalisadores foi determinada por EDX como mostra a Tabela 1. O valor da fração de cobalto encontrado é próximo ao valor nominal para todos os catalisadores. Contudo, o valor da fração de cobalto dos catalisadores encontrado pela técnica de XPS foi menor do que o valor encontrado pela análise de EDX como mostra a Tabela 1, com exceção ao catalisador $1 \mathrm{Co} / \mathrm{Al}_{2} \mathrm{O}_{3}$, que será explicado posteriormente. Para os demais catalisadores, este resultado pode ser explicado, em parte, devido a energia cinética do elétron emitido pelo cobalto, $705,5 \mathrm{eV}$, ser menor do que a energia do cinética do elétron emitido pelo alumínio, $1412 \mathrm{eV}$. Elétrons com maior energia cinética apresentam maior percurso livre médio. Desta forma, uma maior quantidade de elétrons emitidos pela alumina chegará ao detector.

De acordo com Hilmen et al. (1999), a energia de ligação do pico Co2 $\mathrm{p}_{3 / 2}$ é encontrado em $780,2 \mathrm{eV}$ para uma amostra pura de $\mathrm{Co}_{3} \mathrm{O}_{4}$. Para uma amostra de $\mathrm{CoO}$ pura, J. E. Herrera et al. (2001) observaram uma energia de ligação de 780,5 eV para o pico Co2 $\mathrm{p}_{3 / 2}$. Como estas espécies de cobalto apresentam aproximadamente as mesmas energias de ligação, a separação dos picos pode se tornar imprecisa dificultando a quantificação destas espécies em um catalisador. Entretanto, a intensidade dos picos satélites do $\mathrm{Co} 2 \mathrm{p}_{3 / 2}$ das amostras $\mathrm{Co}_{3} \mathrm{O}_{4}$ e $\mathrm{CoO}$ são bem diferentes. A intensidade do pico satélite da amostra $\mathrm{Co}_{3} \mathrm{O}_{4}$ é bem inferior em relação ao pico satélite da amostra $\mathrm{CoO}$ e centralizada a $10 \mathrm{eV}$ acima do pico $\mathrm{Co} 2 \mathrm{p}_{3 / 2}$. O pico satélite da amostra pura de $\mathrm{CoO}$ é encontrado a $5.9 \mathrm{eV}$ acima do pico Co2 $\mathrm{p}_{3 / 2}$ Brundle, (1976). Em nossos experimentos, o pico satélite só foi observado para o espectro do catalisador $1 \mathrm{Co} / \mathrm{Al}_{2} \mathrm{O}_{3}$ e este está localizado 5,5 eV do pico principal, veja Figura 1(a).

$\mathrm{O}$ pico $\mathrm{Co} 2 \mathrm{p}_{3 / 2}$ do aluminato de cobalto é localizado em energias de ligação superiores, 782,0 $\mathrm{eV}$, em relação ao $\mathrm{Co}_{3} \mathrm{O}_{4}$ e $\mathrm{CoO}$. Desta forma, é possível separar o pico referente ao aluminato de cobalto das demais espécies de carbono. Deste modo, a quantidade de $\mathrm{CoAl}_{2} \mathrm{O}_{4}$ presente na superfície foi estimada pela deconvolução do pico $\mathrm{Co} 2 \mathrm{p}_{3 / 2}$ em duas curvas GaussianLorentzain centradas em 780,0 eV referentes ao $\mathrm{CoO}$ e $\mathrm{Co}_{3} \mathrm{O}_{4}$ e, 782,0 referente ao $\mathrm{CoAl}_{2} \mathrm{O}_{4}$. A Tabela 1 mostra a quantidade de aluminato de cobalto presente nos catalisadores.

Pela Figura 1(b) é possível perceber que um aumento na concentração de cobalto nos catalisadores leva a um deslocamento do pico para regiões de mais baixa energia e uma diminuição do pico satélite. O primeiro efeito mostra que há um aumento na quantidade de $\mathrm{Co}_{3} \mathrm{O}_{4}$ e/ou $\mathrm{CoO}$ em relação a quantidade de aluminato de cobalto. $\mathrm{O}$ segundo efeito mostra que há um aumento das espécies $\mathrm{Co}_{3} \mathrm{O}_{4}$ em relação ao $\mathrm{CoO}$. Deste modo é possível concluir que à medida 
que o teor de cobalto aumenta nos catalisadores a fração de cobalto como aluminato vai diminuindo e a fração de $\mathrm{Co}_{3} \mathrm{O}_{4}$ vai aumentando. Analisando a fração de $\mathrm{CoAl}_{2} \mathrm{O}_{4}$ nos catalisadores foi possível observar que há um aumento na quantidade de aluminato de cobalto em função do aumento do teor de cobalto nos catalisadores até a quantidade máxima de 2,1\% no catalisador com 3 e $4 \%$ de cobalto. (Quantidade de $\mathrm{CoAl}_{2} \mathrm{O}_{4}=$ Fração de Co como $\mathrm{CoAl}_{2} \mathrm{O}_{4} \mathrm{x}$ porcentagem de Co presente no catalisador).

Além de identificar os estados de oxidação das espécies químicas, a análise de XPS também permite estimar a dispersão de partícula. Kerkhof e Moulijn (1979) mostraram que a razão entre as intensidades dos elétrons que provem da fase suportada e do suporte está relacionada com a dispersão da fase suportada. Neste modelo é considerado que a fase dispersa está igualmente distribuída na parte interna e externa do suporte e que as partículas apresentam forma cúbica. Desta forma, o tamanho das partículas de cobalto pode ser estimado através da razão entre as intensidades dos picos Co2 $\mathrm{p}_{3 / 2}$ e $\mathrm{Al} 2 \mathrm{p},\left(\mathrm{I}_{\mathrm{Co}} / \mathrm{I}_{\mathrm{Al}}\right)_{\text {cristal }}$, de acordo com a Equação 1:

$$
\frac{\left(\frac{I_{C o}}{I_{A l}}\right)_{\text {cristal }}}{\left(\frac{I_{C O}}{I_{A l}}\right)_{\text {monocamada }}}=\frac{1-e^{\alpha}}{\alpha}
$$

onde $\alpha=\mathrm{C} / \lambda_{\text {Co }}$ C é o tamanho da aresta da partícula e $\lambda_{\text {Co }}$ é o livre caminho médio dos fotoelétrons do cobalto através do óxido de cobalto. A intensidade da monocamada de Co foi calculada usando o modelo de Kerkhof e Moulijn, Equação 2:

$$
\left(\frac{I_{\text {metal }}}{I_{\text {support }}}\right)_{\text {monocamada }}=\left(\frac{n_{C o}}{n_{A l}}\right)_{\text {bulk }} \frac{D\left(\epsilon_{p}\right)}{D\left(\epsilon_{s}\right)}\left(\frac{\sigma_{C o}}{\sigma_{A l}}\right) \frac{\beta_{1}\left(1+e^{-\beta_{2}}\right)}{2\left(1-e^{-\beta_{2}}\right)}
$$

onde $\beta 1=\mathrm{t} / \lambda_{(\mathrm{Al} / \mathrm{Al})}$ e $\beta 2=\mathrm{t} / \lambda_{(\mathrm{Co} / \mathrm{Al})}$. $\lambda_{(\mathrm{Al} / \mathrm{Al})}$ é o livre caminho médio dos fotoelétrons do alumínio através da alumina e $\lambda_{\mathrm{Co} / \mathrm{Al}}$ é o livre caminho médio dos fotoelétrons do cobalto atravessando a alumina. O termo $\left(\mathrm{n}_{\mathrm{Co}} / \mathrm{n}_{\mathrm{Al}}\right)$ bulk é a razão atômica entre a quantidade global de cobalto e alumínio dos catalisadores. O livre caminho médio dos fotoelétrons foi obtido por Penn (1976). $\mathrm{D}(\varepsilon)$ é a eficiência do detector e $\sigma$ é a seção de choque. Estes parâmetros foram substituídos pelo fator de sensibilidade atômico do software Avantage, 12,62 para o Co2 $\mathrm{p}_{3 / 2}$ e 0,537 for Al2p.

Enquanto as análises de EDX fazem medidas da composição global dos catalisadores, as análises de XPS fazem a medida da composição superficial dos catalisadores. Tanto a composição global quanto a superficial apresentaram o mesmo valor para o catalisador $1 \mathrm{Co} / \mathrm{Al}_{2} \mathrm{O}_{3}$, como mostra a Tabela 1. Isto indica que cobalto está igualmente distribuído por todo catalisador. Além disto, a razão entre as áreas dos picos $\operatorname{Co} 2 \mathrm{p}_{3 / 2}$ e $\mathrm{A} 12 \mathrm{p}$ medidas pelo XPS é maior que a razão calculada para uma monocamada de cobalto como mostra a Tabela 2. Isto significa que o cobalto está altamente disperso, mas não segue o modelo de Kerkhof e Moulijn. Esta alta dispersão pode ser explicada pela migração dos átomos de cobalto para o interior da matriz da alumina como observado por Liotta (2003). A Figura 2 (b) representa a dispersão do cobalto como no modelo proposto por Kerkhof e Moulijn e a Figura 2(a) mostra o esquema de dispersão do cobalto como descrito Liotta et al. (2003). Na Figura 2, os quadrados pretos representam as partículas de cobalto e as setas pretas e cinzas representam os elétrons escapando dos átomos de cobalto e alumínio respectivamente. 
Tabela 2 - Intensidades do espectro de XPS dos catalisadores, $\left(\mathrm{I}_{\mathrm{Co}} / \mathrm{I}_{\mathrm{Al}}\right)_{\text {crist }} /\left(\mathrm{I}_{\mathrm{Co}} / \mathrm{I}_{\mathrm{Al}}\right)_{\text {mono; }}$ tamanho médio das partículas de óxido de cobalto $\left(\mathrm{CoO}\right.$ e $\left.\mathrm{Co}_{3} \mathrm{O}_{4}\right)$ calculado pelo modelo de Kerkhof e Moulijn, $\mathrm{d}_{\mathrm{XPS}}$; tamanho do cristalito de $\mathrm{Co}_{3} \mathrm{O}_{4}$ determinado por XRD e; fração de Co como $\mathrm{Co}_{3} \mathrm{O}_{4}$ determinado por TPR

\begin{tabular}{|c|c|c|c|c|c|}
\hline Amostra & $\begin{array}{c}\left(\mathrm{I}_{\mathrm{Co}} / \mathrm{I}_{\mathrm{Al}}\right)_{\text {crist }} / \\
\left(\mathrm{I}_{\mathrm{Co}} / \mathrm{I}_{\mathrm{Al}}\right)_{\text {mono }}\end{array}$ & $\mathrm{d}_{\mathrm{XPS}}(\mathrm{nm})$ & $\mathrm{d}_{\mathrm{XRD}}(\mathrm{nm})$ & $\begin{array}{c}\text { Grau de } \\
\text { redução }(\%)\end{array}$ & $\begin{array}{c}\text { Fração de } \\
\mathrm{Co}_{3} \mathrm{O}_{4}\end{array}$ \\
\hline $1 \mathrm{Co} / \mathrm{Al}_{2} \mathrm{O}_{3}$ & 1,3 & - & - & 0 & 0,00 \\
\hline $2 \mathrm{Co} / \mathrm{Al}_{2} \mathrm{O}_{3}$ & 0,3 & 2 & - & 27 & 0,07 \\
\hline $3 \mathrm{Co} / \mathrm{Al}_{2} \mathrm{O}_{3}$ & 0,2 & 4 & 10 & 30 & 0,16 \\
\hline $4 \mathrm{Co} / \mathrm{Al}_{2} \mathrm{O}_{3}$ & 0,2 & 4 & 14 & 31 & 0,24 \\
\hline
\end{tabular}

A intensidade dos elétrons que escapam do catalisador é função da profundidade em relação à superfície. Aplicando a lei de Lambert-Beer temos $\mathrm{I}(\mathrm{z})=\mathrm{I}_{0} \mathrm{e}^{-\mathrm{z} / \lambda}$. Onde $\mathrm{I}_{0}$ é a intensidade dos elétrons na superfície e $\mathrm{z}$ é a profundidade. Na Figura 2 a intensidade dos elétrons que deixam o cobalto é representada pela curva preta enquanto a intensidade dos elétrons que deixam o alumínio é representada pela curva cinza.
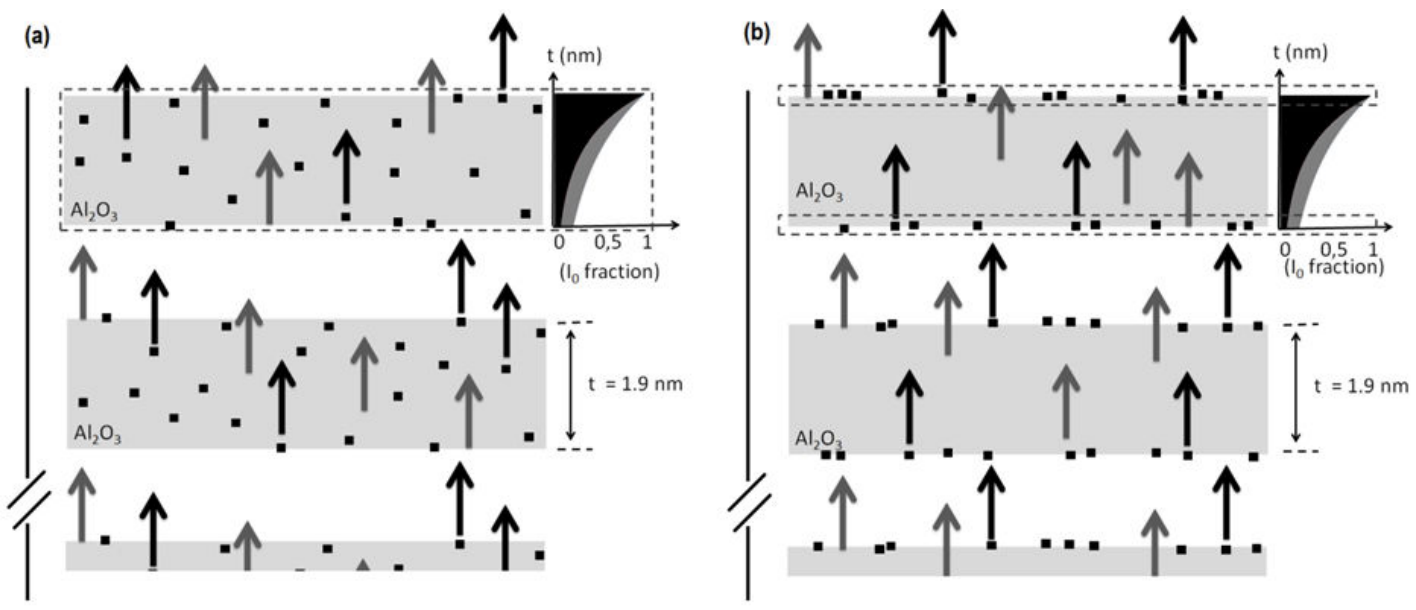

Figura 2 - Modelos de catalisadores. (a) dispersão homogênea do cobalto por todo suporte como descrito por Liota et al, (b) dispersão do cobalto como no modelo de Kerkhof e Moulijn

Aplicando a lei de Lambert-Beer e para a primeira camada de cada modelo da Figura 2, é encontrado que a intensidade dos elétrons que deixam o cobalto no modelo de Liota et al. é $23 \%$ maior em relação ao modelo de Kerkhof e Moulijn. Como o catalisador $1 \mathrm{Co} / \mathrm{Al}_{2} \mathrm{O}_{3}$ apresentou alta razão entre as intensidade $\mathrm{Co} 2 \mathrm{p}_{3 / 2} / \mathrm{A} 12 \mathrm{p}$ há um indicativo de que o cobalto está disperso como no modelo descrito por Deganello.

Os catalisadores com maior quantidade de cobalto, 2, 3 e 4\% de cobalto, apresentaram valores baixos para a razão $\left(\mathrm{I}_{\mathrm{Co}} / \mathrm{I}_{\mathrm{Al}}\right)_{\text {cristal }} /\left(\mathrm{I}_{\mathrm{Co}} / \mathrm{I}_{\mathrm{Al}}\right)_{\text {mono }}$ e o modelo de Kerkhof e Moulijn pode ser aplicado. $\mathrm{O}$ tamanho de partícula calculado representa o tamanho de partícula médio das espécies de óxido cobalto, $\mathrm{CoO}$ e $\mathrm{Co}_{3} \mathrm{O}_{4}$. $\mathrm{O}$ tamanho médio destas partículas pode ser observado na Tabela 2.

As analises de XRD foram usadas para determinar as fases cristalinas e estimar o diâmetro 
dos cristalitos de óxidos de cobalto. A Figura 3 mostra os difratogramas do suporte e dos catalisadores. Os picos em 40,0 e $66,7^{\circ}$ estão relacionados à fase gama da alumina e podem ser observados em todas as amostras. Os catalisadores com 3 e $4 \%$ de cobalto apresentaram picos de difração em $31,3,36,9$ e $59,5^{\circ}$ e estes picos podem estar relacionados a presença de $\mathrm{Co}_{3} \mathrm{O}_{4}$ ou $\mathrm{CoAl}_{2} \mathrm{O}_{4}$. Deste, modo não foi possível distinguir por XRD estas fases. Entretanto, a análise de XPS mostrou que o aluminato de cobalto está bem disperso e que existe um máximo em sua formação, 2,1\% em peso no catalisador 3 e $4 \mathrm{Co} / \mathrm{Al}_{2} \mathrm{O}_{3}$. Desta forma, o aumento da intensidade do pico de difração do catalisador com $4 \%$ de cobalto em relação ao catalisador com $3 \%$ não pode ser atribuído a espécie $\mathrm{CoAl}_{2} \mathrm{O}_{4}$. Deste modo, os picos de difração foram atribuídos às espécies $\mathrm{Co}_{3} \mathrm{O}_{4}$. Os picos de difração do $\mathrm{CoO}, 42,6$ e $61,8^{\circ}$, não foram observados nos difratogramas. Isto pode ocorrer devido a sua baixa concentração, ao seu pequeno diâmetro ou apresentar-se na forma amorfa. $\mathrm{O}$ diâmetro médio dos cristalitos de $\mathrm{Co}_{3} \mathrm{O}_{4}$ foram calculadas a partir do pico à $36,9^{\circ}$ usando a equação de Scherrer. Estes resultados podem ser observados na Tabela 2.

O diâmetro médio dos cristalitos encontrado pelas análises de XRD são maiores que os diâmetros de partícula encontrados pelas análises de XPS. Nas análises de XPS, o diâmetro das partículas foi estimado pelos elétrons que deixam as espécies $\mathrm{CoO}$ e $\mathrm{Co}_{3} \mathrm{O}_{4}$. Desta forma, o diâmetro encontrado por XPS é um diâmetro médio destas partículas. Como os picos de difração da fase $\mathrm{CoO}$ não foram observados e os de $\mathrm{Co}_{3} \mathrm{O}_{4}$ foram observados nas análises de XRD é sugerido que as partículas de $\mathrm{CoO}$ sejam menores que as partículas de $\mathrm{Co}_{3} \mathrm{O}_{4}$.
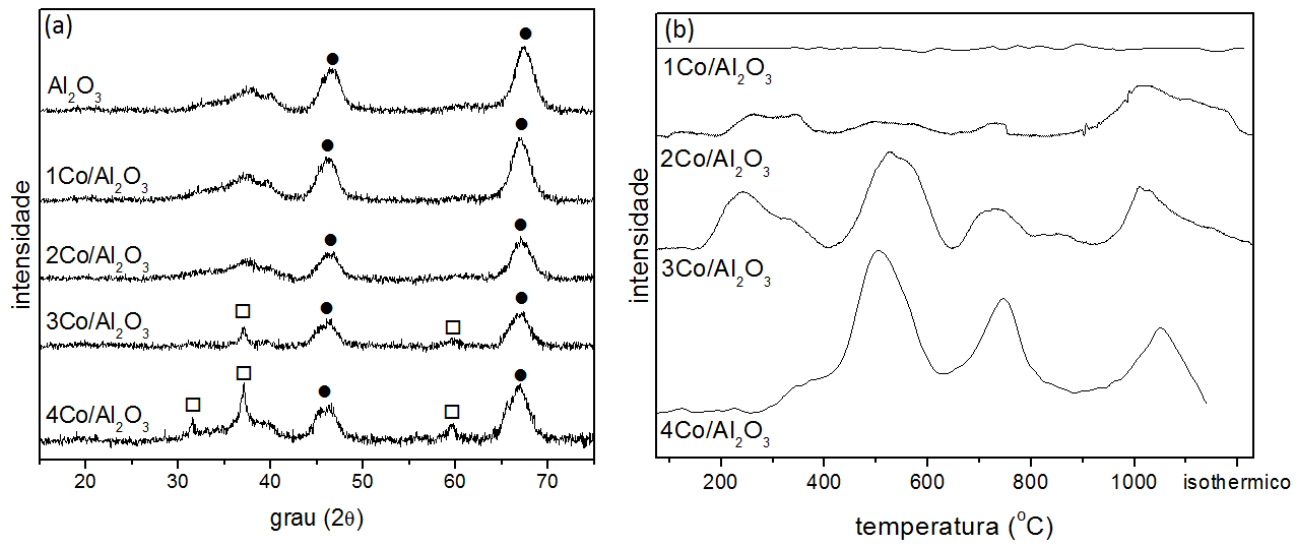

Figura 3(a) - Difração de raios-X dos catalisadores: $\square \mathrm{Co}_{3} \mathrm{O}_{4}$, $\bullet$ gamma alumina. (b) perfil de redução dos catalisadores

As análises de TPR pode ajudar a identificar e quantificar as espécies de cobalto em catalisadores de suportados em alumina. $\mathrm{O}$ perfil de redução do catalisador $1 \mathrm{Co} / \mathrm{Al}_{2} \mathrm{O}_{3}$ não apresentou consumo de hidrogênio como mostra a Figura 3 (b). Isto demonstra que somente espécies de difícil redução como $\mathrm{CoAl}_{2} \mathrm{O}_{4}$ e $\mathrm{CoO}$ com forte interação com o suporte estão presentes neste catalisador. As análises de XPS mostraram que 93\% destas espécies na superficie são aluminato de cobalto. A presença destas espéciese em um catalisador normalmente não são desejadas pois, em grande parte, o cobalto deve estar em sua fase metálica para catalisar a reação.

$\mathrm{O}$ perfil do catalisador $2 \mathrm{Co} / \mathrm{Al}_{2} \mathrm{O}_{3}$ mostrou quatro pequenos picos de redução. $\mathrm{O}$ pico à temperatura de $364{ }^{\circ} \mathrm{C}$ é atribuido a redução do óxido de cobalto amorfo com propiedades redox semelhantes ao $\mathrm{Co}_{3} \mathrm{O}_{4}$. O pico a temperatura de $507{ }^{\circ} \mathrm{C}$ é atribuido a redução em duas etapas do $\mathrm{Co}_{3} \mathrm{O}_{4}$. O pequeno pico a $737{ }^{\circ} \mathrm{C}$ está asociado à redução do $\mathrm{CoO}$ com forteinteração com a 


\section{9 a 22 de outubro de 2014 \\ Florianópolis/SC}

alumina e, o pico a $1000{ }^{\circ} \mathrm{C}$ está relacionado a redução do aluminato de cobalt. O perfil de redução do catalizador $3 \mathrm{Co} / \mathrm{Al}_{2} \mathrm{O}_{3}$ também mostrou a redução de quatro espécies de cobalto diferentes como no catalizador $2 \mathrm{Co} / \mathrm{Al}_{2} \mathrm{O}_{3}$. Contudo, a fração de cada espécie reduzida foi diferente. A fração de $\mathrm{Co}_{3} \mathrm{O}_{4}$, pico a $507{ }^{\circ} \mathrm{C}$, foi mais elevada no catalisador com $3 \%$ de cobalto do que no com $2 \%$. A intensidade deste pico ficou ainda maior para o catalizador $4 \mathrm{Co} / \mathrm{Al}_{2} \mathrm{O}_{3}$.

O grau de redução é um importante parâmetro para calcular a atividade catalítica. A Tabela 2 mostra o grau de redução dos catalisadores. É possível observar que um aumento no teor metálico dos catalisadores fez com que o grau de redução também aumenta-se. Contudo, há uma grande fração de cobalto que não foi reduzida. Enquanto todo $\mathrm{Co}_{3} \mathrm{O}_{4}$ é reduzido a sua forma metálica, somente uma pequena fração do $\mathrm{CoO}$ e do $\mathrm{CoAl}_{2} \mathrm{O}_{4}$ foi reduzido até a temperatura de $1000{ }^{\circ} \mathrm{C}$. Desta forma, não foi possível determinar por TPR a fração de $\mathrm{CoO}$ e $\mathrm{CoAl}_{2} \mathrm{O}_{4}$ presente nos catalisadores. Entretanto, se considerarmos a fração de $\mathrm{CoAl}_{2} \mathrm{O}_{4}$ na superficie do catalisador, determinado por XPS, aproximadamente igual a fração de $\mathrm{CoAl}_{2} \mathrm{O}_{4}$ presente em todo catalisador, é possível estimar a fração de $\mathrm{CoO}$ dos catalisadores.

A Figura 4 mostra a dirtribuição das espécies de cobalto nos catalisadores. As colunas que apresentam um quadrado em seu topo representam a fração das espécies de cobalto que foram reduzidas na análise de TPR e as colunas com a losango em seu topo representam as espécies de cobalto que não foram reduzidas durante a análise de TPR.
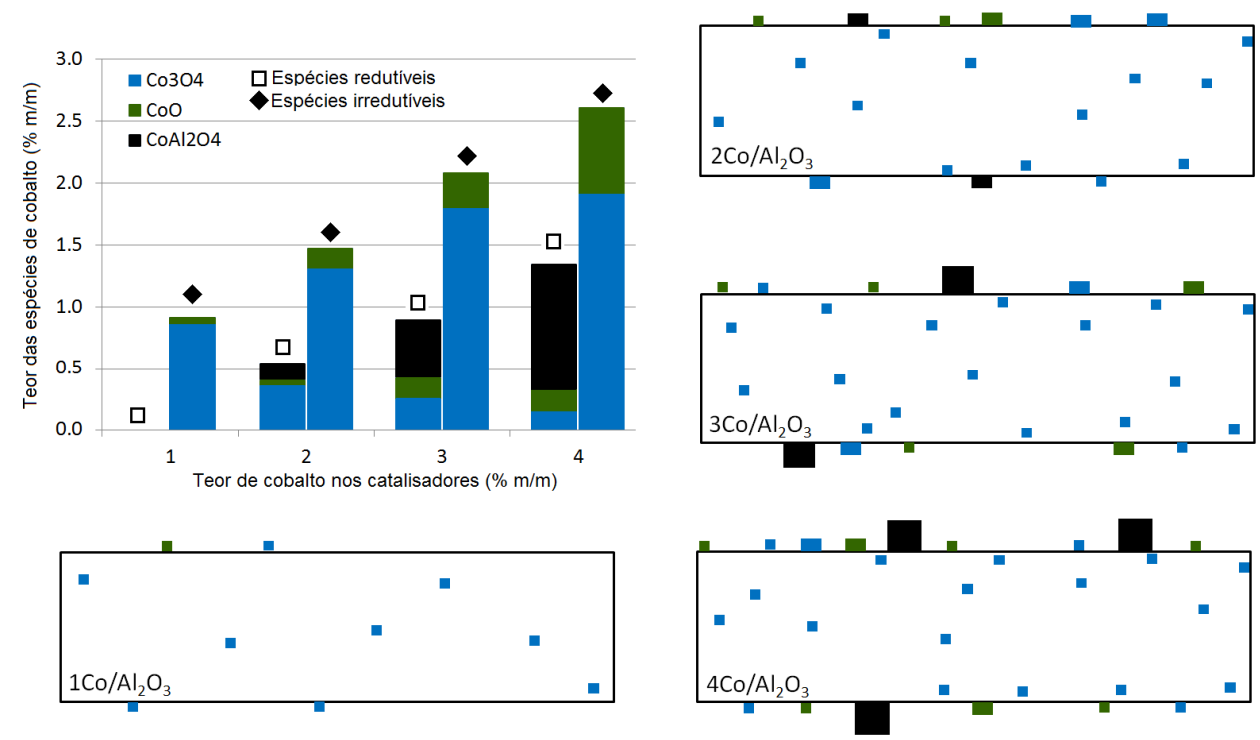

Figura 4. Distribuição das espécies de cobalto nos catalisadores. 口espécies que foram reduzidas na análise de TPR; $\diamond$ e espécies que não foram reduzidas na análise de TPR. Esquema da distribuição das espécies de cobalto nos catalisadores

Com os resultados das caracterizações foi feito um esquema da distribuição das espécies de cobalto presentes nos catalisadores como mostra a Figura 4. Para o catalisador $1 \mathrm{Co} / \mathrm{Al}_{2} \mathrm{O}_{3}$, só existem espécies irredutíveis como demostrado por TPR e, estas espécies são, em grande parte, $\mathrm{CoAl}_{2} \mathrm{O}_{4}$ que estão altamente dispersos por todo o suporte como mostrou a técnica de XPS. Este aluminato de cobalto altamente disperso é representando por pequenos quadrados azuis. Para o catalisador de $2 \mathrm{Co} / \mathrm{Al}_{2} \mathrm{O}_{3}$, além da presença do aluminato de cobalto altamente disperso, a análise de TPR indicou a presença de espécies redutíveis como $\mathrm{Co}_{3} \mathrm{O}_{4}, \mathrm{CoO}$ e $\mathrm{CoAl}_{2} \mathrm{O}_{4}$, representadas 


\section{9 a 22 de outubro de 2014 \\ Florianópolis/SC}

pelos retângulos pequenos na superfície do suporte. Estas partículas devem estar bem dispersas pois não foi possível detecta-las nas análises de XRD. No catalisador $3 \mathrm{Co} / \mathrm{Al}_{2} \mathrm{O}_{3}$ foi possível deterctar por XRD a presença de $\mathrm{Co}_{3} \mathrm{O}_{4}$. Estas partículas maiores são representadas pelos quadrados pretos nos catalisadores com 3 e $4 \%$ de cobalto.

\section{CONCLUSÃO}

As técnicas de fissisorção, EDX, XPS, DRX e TPR permitiram a identificação das diferentes espécies de cobalto presentes em catalisadores suportados em $\gamma$-alumina e estimar a dispersão destas espécies. O aluminato de cobalto está altamente disperso em todas as amostras e, com o aumento do teor de cobalto nos catalisadores a fração de cobalto como aluminato diminui enquanto a fração de $\mathrm{CoO}$ e $\mathrm{Co}_{3} \mathrm{O}_{4}$ aumentam assim como o grau de redução. $\mathrm{O}$ diâmetro das partículas de $\mathrm{Co}_{3} \mathrm{O}_{4}$ foram 10 e $14 \mathrm{~nm}$ para os catalisadores com 3 e $4 \%$ de cobalto, respectivamente. Para os demais catalisadores, os cristalitos de $\mathrm{CoO}$ e $\mathrm{Co}_{3} \mathrm{O}_{4}$ não foram detectadas por XRD. Contudo, a estimativa do tamanho médio do diâmetro dos óxidos de cobalto por XPS para o catalisador $2 \mathrm{Co} / \mathrm{Al}_{2} \mathrm{O}_{3}$ foi de $2 \mathrm{~nm}$ enquanto para o catalisador com $1 \%$ de cobalto as partículas estão $100 \%$ dispersas.

\section{REFERÊNCIAS}

BRUNDLE, C.R.; CHUANG, T.J.; RICE, D.W. X-ray photoemission study of the interaction of oxygen and air with clean cobalt surfaces. Surface Science v. 60, p. 286-300, 1976.

CHEUNG, C.L; KURTZ, A; PARK, H; LIEBER, C.M. Diameter-controlled synthesis of carbon nanotubes. J. of physical chemistry b v. 106, p.2429-2433, 2002.

DEGANELLO, G.; LIOTTA, L.F.; $\mathrm{CoO}_{\mathrm{x}}$ catalysts supported on alumina and alumina-baria: influence of the support on the cobalt species and their activity in NO reduction by $\mathrm{C}_{3} \mathrm{H}_{6}$ in lean conditions. Appl. Cat. A: G v. 245 p. 167-177, 2003.

HILMEN, A.M.; SCHANKE, D.; HANSSEN, K.F.; HOLMEN, A. Study of the effect of water on alumina supported cobalt. Appl. Cat. A: General, v.186, p.169-188, 1999.

HERRERA, J.E.; ALVAREZ, W.E.; RESASCO, D.E. Relationship between the Structure/Composition of Co-Mo Catalysts and Their Ability to Produce Single-Walled Carbon Nanotubes by CO Disproportionation J. of Catalysis, v.204, p.129-145, 2001.

IIJIMA, S. Helical microtubules of graphitic carbon. Nature v. 56, p.354, 1991.

KERKHOF, F.P.J.M.; MOULIJN, J.A. Quantitative Analysis of XPS Intensities for Supported Catalysts. J.Phys. Chem. v. 83, pag.1612-1619, 1979.

LIOTTA, L.F.; MACALUSO, A.; DI CARLO, G.; DEGANELLO, G. CoOx catalysts supported on alumina and alumina-baria: influence of the support on the cobalt species and their activity in NO reduction by $\mathrm{C}_{3} \mathrm{H}_{6}$ in lean conditions. Appl. Catal. A: General v. 245, p.167, 2003

LI, N; WANG, X; DERROUICHE, S, Role of Surface Cobalt Silicate in Single-Walled Carbon Nanotube Synthesis from Silica-Supported Cobalt Catalysts. ACS Nano v. 4, p. 1759-1767, 2010.

PENN, D.R. Quantitative chemical analysis by ESCA. J. Elect. Spectr.Rel. Phen., v.9 p 29-40, 1976.

ZHANG, J.L; SUN, Y.H. Chemical treatment of gama $\mathrm{Al}_{2} \mathrm{O}_{3}$ and its influence on the properties of Cobased catalysts for Fischer-Tropsch synthesis. Appl. Cat. a-general v. 243 p. 121-133, 2003. 